\title{
A Stimulus-Oriented Approach to Memory
}

\author{
Robert Sekuler $^{1}$ and Michael J. Kahana ${ }^{2}$ \\ ${ }^{1}$ Volen Center for Complex Systems, Brandeis University, and ${ }^{2}$ Department of Psychology, University of Pennsylvania
}

\begin{abstract}
Our understanding of short-term recognition memory can be enhanced by careful choice and control of test materials. Theory-driven manipulation of memory test stimuli, including visual textures, human faces, and complex sounds, minimize individual differences and make it possible to predict recognition performance for specific combinations of stimulus items. This stimulus-oriented approach to memory reveals that stimulus similarity plays two different important roles in recognition memory. By exploiting tools used in psychophysics, it is possible to generate mnemometric functions-detailed "snapshots" that capture key features of subjects' memory strength.
\end{abstract}

KEYWORDS-recognition; short-term memory; models; criterion; decision making; memory

In his monumental Handbook of Experimental Psychology, S.S. Stevens (1951, p. 31) observed "In a sense there is only one problem of psychophysics, namely the definition of the stimulus. In this same sense there is only one problem in all of psychology—and it is the same problem."

Careful attention to stimulus choice, design, and control has led to many of the key insights of sensory research. We believe that a comparable, stimulus-oriented approach to memory affords equally significant theoretical leverage. This belief stimulated our own collaboration, which draws on sensory research (R.S.) as well as memory research (M.J.K.). The work described in this article illustrates a few of the benefits that can come from a stimulus-oriented approach to memory. In particular, knowing the similarity relationships among memory-test materials opens new ways to examine theoretical propositions about memory.

We do not claim to be the only researchers who appreciate the connections between memory and perception. Others have recently recognized memory's role in even the most basic perceptual tasks. This recognition makes particularly good sense,

Address correspondence to Robert Sekuler, Volen Center for Complex Systems, Brandeis University, Waltham, Massachusetts 01742; e-mail: sekuler@brandeis.edu. as some contemporary models of memory share assumptions with models of sensory discrimination. But when memory models fail to link their stimulus representations to measures of perceptual similarity, they needlessly limit their ability to account for a variety of important phenomena.

To examine short-term memory, we use test stimuli that are little burdened by the complexities of extra-laboratory associations. Unlike semantically rich items such as words or images of objects that are easily categorized or named, our stimuli resist symbolic coding and mediation. These stimuli include visual textures (gratings, also known as Gabor patches). The top row of Figure 1 shows samples of these stimuli, which are constructed by combining gratings of differing orientations and/or spatial frequencies (number of bars per degree of visual angle). Small changes in spatial frequency subtly alter the appearance of the stimuli. Controlled variation in test materials allows memory and decision processes to be manipulated as some particular theoretical goal requires. This facilitates tests of theoretical claims about how memory's contents are expressed in recognition judgments. In our experiments, the similarity relations among stimuli are designed to challenge correct recognition and to produce errors, which are rich fodder for modeling. In addition, stimuli can be tailored to each subject's own sensory-discrimination capacity. This limits the ability of sensory function to exaggerate differences in memory function from one individual to another.

\section{GLOBAL MATCHING MODELS}

In one common recognition task, a series of study items is presented at the start of each trial. This series is followed, after a short retention interval, by a probe item. The subject's task is to decide whether this probe $(\mathrm{P})$ replicates any of the study items just seen. On some trials, called target trials, $\mathrm{P}$ replicates one of the study items; on the remaining trials, called lure trials, P replicates none of the study items. Early models of recognition memory assumed that the receipt of the probe produced some brain signal of that stimulus' familiarity. This strength of that signal could then be used to distinguish novel from repeated 

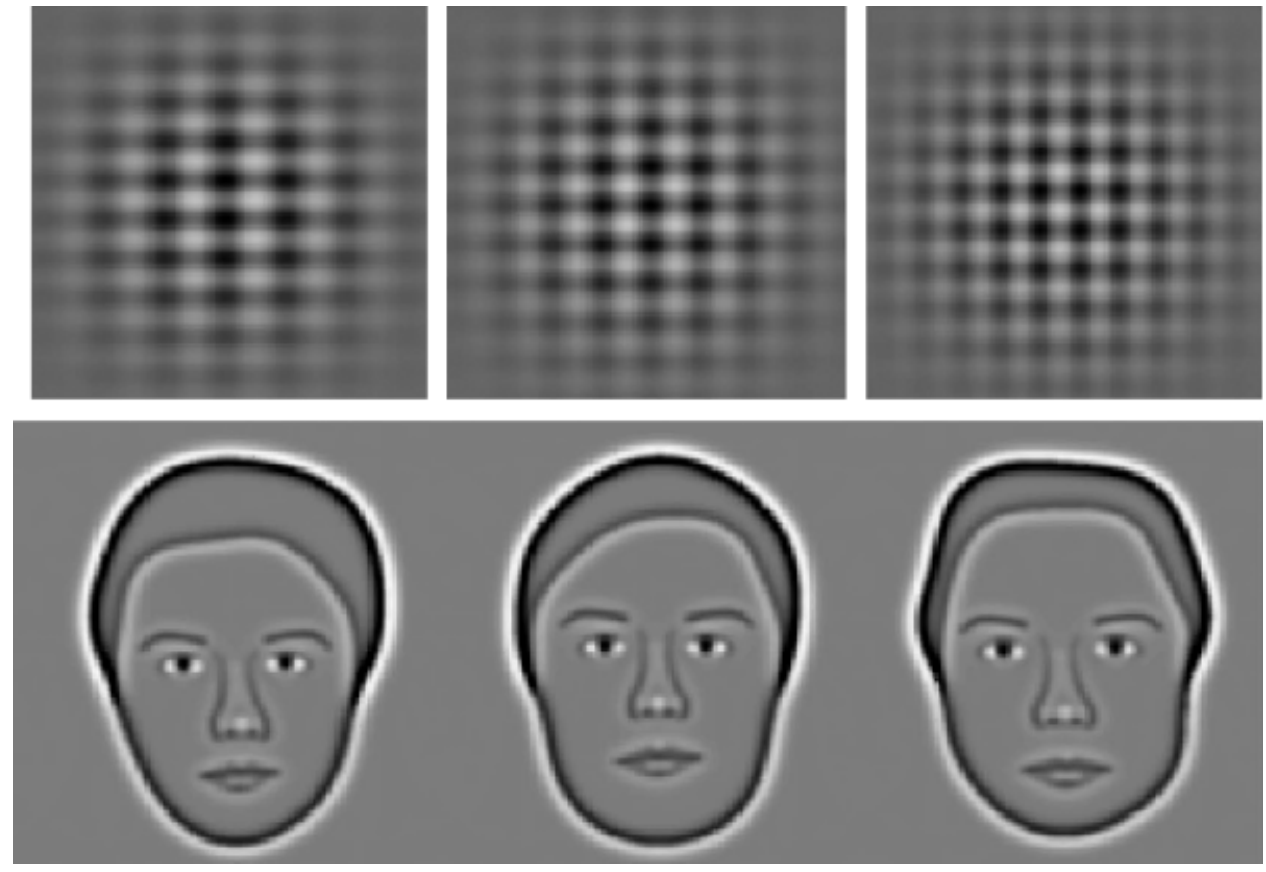

Fig. 1. Examples of stimuli for our experiments. Top row: three compound gratings (Gabor patches) in which both horizontal and vertical spatial frequencies (number of bars) increase by about $15 \%$ between adjacent gratings. Bottom row: Sample synthetic faces used in stimulus-oriented study of memory. Each of these exemplars is derived from a different real face, and each lies the same metric distance away from a face that is the average of a large set of real faces.

occurrences of an item-that is, to distinguish lure trials from target trials. This traditional account ran smack into two problems, one conceptual and one empirical. Conceptually, it could not explain how, out of all the memories that were stored on a trial, some one critical memory could be isolated and used in generating the familiarity signal. Empirically, the account could not explain why recognition judgments were governed not only by the probe's similarity to the lone matching study item but also by its similarity to other study items.

To overcome both of these problems, global matching models were introduced. Such models assume that a recognition judgment reflects just a single value that summarizes information about the multiple items stored in memory. For example, in our own model, that single value represents "summed similarity," the sum of the probe's similarity to each and every one of the study items.

Figure $2 \mathrm{~A}$ is a diagram of the global matching component of our own model. We assume that each study item generates its own representation or exemplar in memory. Following the tradition of multidimensional signal-detection theory (Ashby \& Maddox, 1998), the representation of any one stimulus varies from occasion to occasion-which is suggested, in Figure 2, by the "clouds" that correspond to each exemplar.

To generate the value of summed similarity, P's similarity to each study item is assessed and those separate assessments are added together. On average, summed-similarity values produced on target trials will exceed ones produced on lure trials (because every target trial, but not every lure trial, will have at least one exemplar that is highly similar to P). Incidentally, researchers have already identified neural circuits capable of carrying out this computation-for example, familiarity-sensitive neurons found in the perirhinal cortex (Bogacz \& Brown, 2003), a region in the medial temporal lobe.

The diagram in Figure 2A shows that once summed similarity has been computed, its value is sent to a decision module, where it is compared against a criterion. The criterion value reflects (a) the perceived probability that the probe would have replicated a study item and (b) the cost of a false recognition along with the value of a correct one. If summed similarity exceeds the criterion, the probe is judged "old" (it replicates one of the study items); otherwise, the probe is judged "new."

\section{FINDING NEMo: STUDY-ITEM HOMOGENEITY}

In our first experiments, using gratings as test materials (Kahana \& Sekuler, 2002), the summed-similarity model shown in Figure $2 \mathrm{~A}$ produced a passable but imperfect account of the results. For some trials in particular, the model's predictions were way offsomething was clearly wrong. To find out what, we examined our results through the lens of the similarity relations among each trial's stimuli. Knowing what these similarity relations were, we identified trials on which recognition performance departed significantly from predictions based on summed similarity alone (Kahana \& Sekuler, 2002). A close examination of the "deviant" 


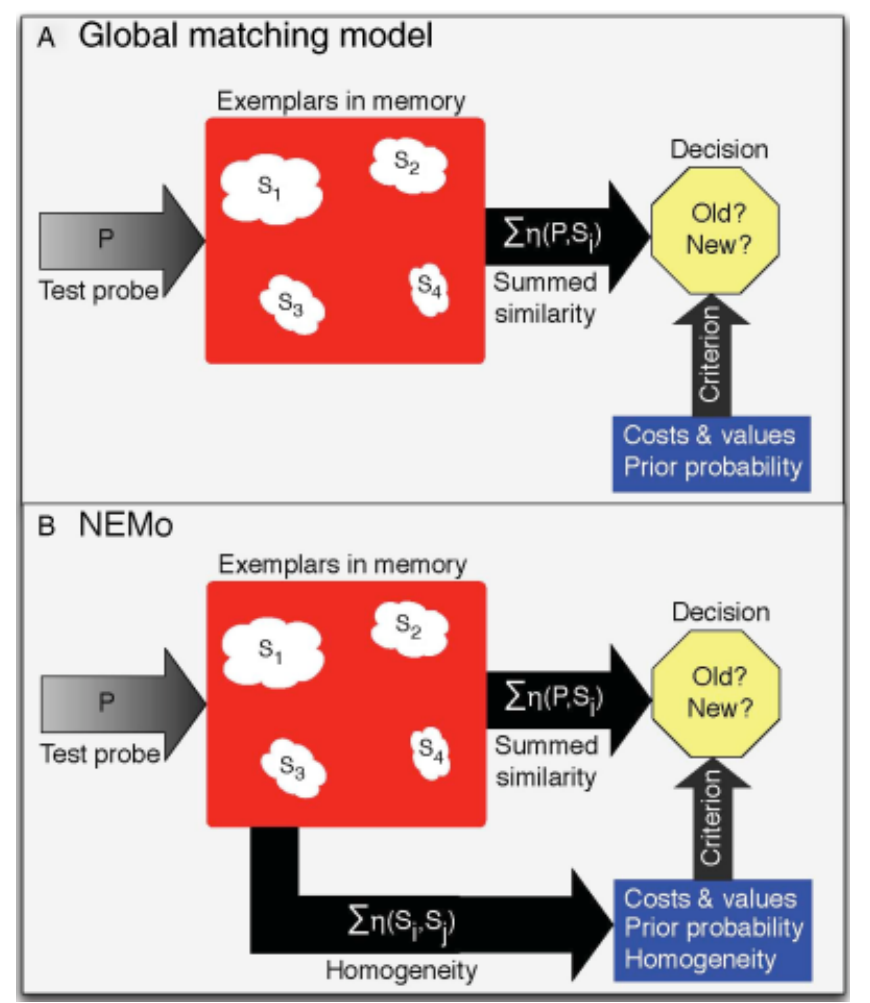

Fig. 2. Schematic diagram illustrating basic components of global matching models (panel A) and NEMo, the Noisy Exemplar Model (panel B). In both models, the presentation of four study items, $S_{1} \ldots S_{4}$, gives rise to exemplars of those items in memory. Also in both models, summed similarity is computed by summing the similarity of the probe $(\mathrm{P})$ to each of the separate memory exemplars. In the global matching model (panel A), the value of summed similarity is compared against a criterion, and the outcome of the comparison determines the subject's response that $\mathrm{P}$ is "old" or "new." In panel B, NEMo assumes that the criterion is influenced by one additional element, stimulus homogeneity, a measure of how similar the memory exemplars are to one another. Once this additional element has entered into the criterion, the process that leads to a decision is the same as in panel A.

trials revealed the operation of a previously hidden, but powerful, influence on recognition performance. And from this discovery, our Noisy Exemplar Model (NEMo) was born.

Reflecting the analysis of deviant trials in our first experiments, Figure 2B highlights NEMo's key new element, an additional influence on the criterion value: the homogeneity of the study items (Nosofsky \& Kantner, 2006). Functionally, the impact of summed similarity is modulated by study-item homogeneity. Empirically, when study items are similar to one another, the criterion value is increased, reducing the likelihood of false recognitions (saying "yes" on lure trials). So with greater homogeneity, subjects demonstrate increased ability not to be fooled into falsely recognizing probes that resemble but do not perfectly match one of the study items. Note that what seems to be an advantage-being fooled less often — comes at a price: reduced likelihood of correct recognitions.

It is important to note that the impact of study-item homogeneity is not restricted to recognition of Gabor patches but has been confirmed with various other stimuli-ones as diverse as color patches (Nosofsky \& Kantner, 2006); realistic, synthetic human faces (see Fig. 1, bottom panel; Yotsumoto, Kahana, Wilson, \& Sekuler, 2007); and abstract, complex sounds (Visscher, Kaplan, Kahana, \& Sekuler, 2007). Note that without a stimulus-oriented characterization of study-item homogeneity, this powerful influence on recognition performance would have gone undetected, lost in the data's unexplained variance. However, by augmenting the basic, summed-similarity framework with the idea that within-list summed similarity influences recognition decisions, NEMo fulfills one goal of any model: shifting substantial variance in the data from the "random" (unexplained) column to the "deterministic" (accounted for) column.

\section{MEMORIES COME, MEMORIES GO (QUICKLY)}

A stimulus-oriented approach to memory also makes it possible to examine the temporal ebb and flow of influences on recognition. For example, Visscher et al. (2007) showed that, in contrast to other determinants of the subject's criterion-which develop over many trials-homogeneity's entire influence develops quickly, within just a single trial, and then dissipates just as quickly. Visscher et al. (2007) measured recognition memory for so-called moving-ripple stimuli-sounds generated by combining many sounds having different frequencies. Such sounds, whose loudness fluctuates regularly over time, have a formal resemblance to most speech sounds.

Visscher et al. (2007) constructed two types of lure trials, ones in which the study items were homogeneous (the study items were only one just-noticeable difference apart) and ones in which the study items were heterogeneous (the study items were at least four just-noticeable differences apart). The former were expected to produce a high criterion value for summed similarity; the latter were expected to produce a low criterion value for summed similarity. The two types of lure trials were intermixed among various other trials. The mixture of trials was adjusted so that identical trials followed either "low criterion" trials (heterogeneous study items) or "high criterion" trials (homogeneous study items).

This portion of Visscher et al.'s (2007) larger study produced two noteworthy results related to false recognitions. First, as NEMo predicts, low-criterion trials produced far higher false-alarm rates than high-criterion trials did. Second, on the immediately succeeding, neutral trials, there was no evidence of the criterion differences that had been generated on the previous trial: False-alarm rates on trials that followed high-criterion trials were no different from false-alarm rates on trials that followed low-criterion trials. So, homogeneity's influence appears to reach full strength during the course of a single trial, as subjects respond to the study items on that trial, and then recedes immediately thereafter. Note that this remarkable encapsulation of criterion change stands in contrast to the intertrial 

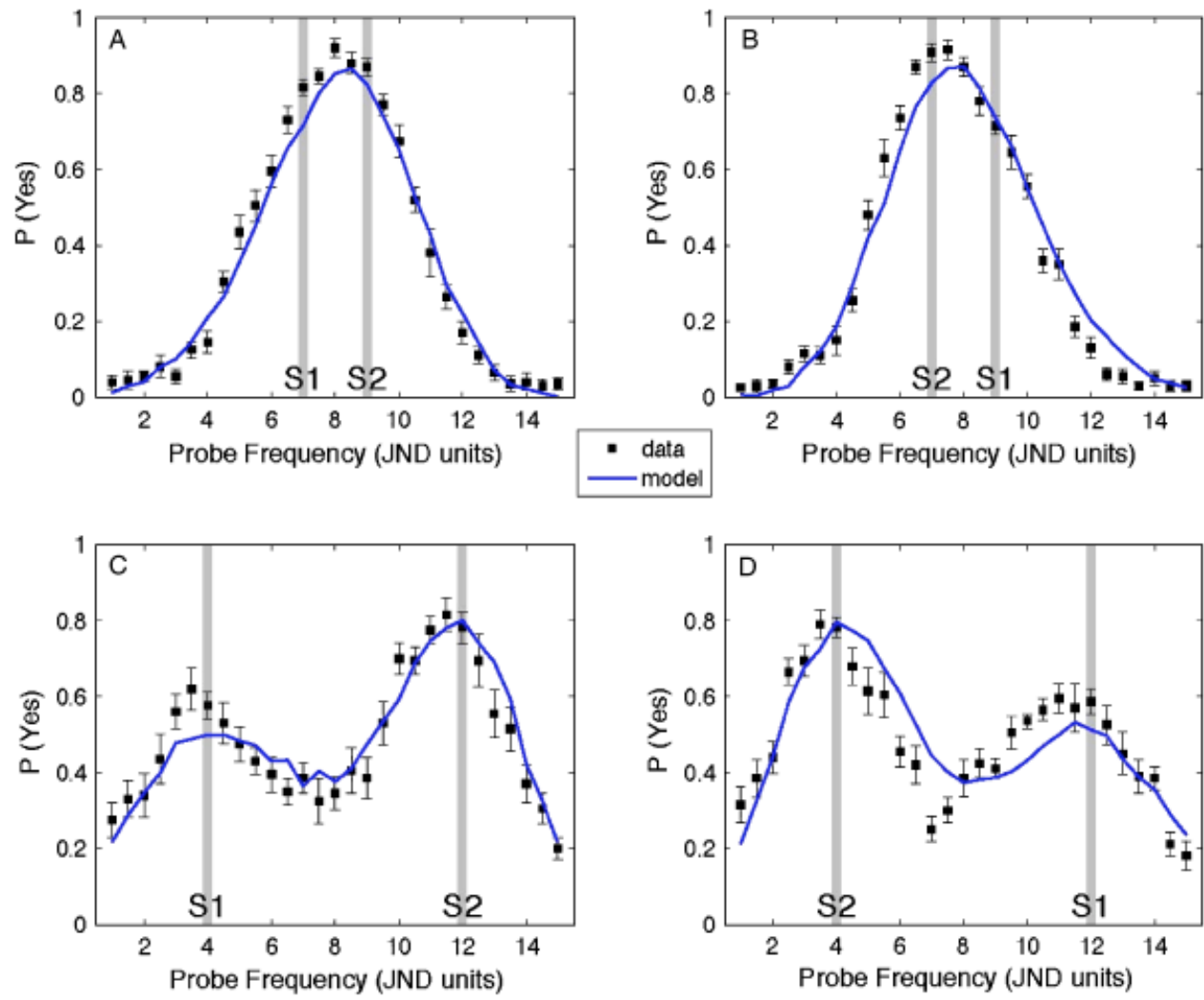

Fig. 3. Mnemometric functions (the proportion of "yes" responses) generated as a probe (P) varied in spatial frequency relative to the frequencies of two study items. Study-item frequencies are shown by the vertical gray lines labeled S1 and S2, signifying the first and second study item, respectively. Panels A and B show mnemometric functions with study items that differed by two just-noticable differences (JNDs); panels C and $D$ show mnemometric functions with study items that differed by eight JNDs. Panels A and C are for trials on which the second study item's spatial frequency was higher than the first study item's; Panels B and D are for the reverse case. The solid blue line in each panel represents the values predicted by our model, NEMo. Adapted from Kahana, Zhou, Geller, \& Sekuler (2007).

proactive interference that has been reported in prior studies with semantically related stimuli.

\section{MNEMOMETRIC FUNCTIONS: SNAPSHOTS OF MEMORY}

For over a century, psychophysical research has exploited a tool called the psychometric function. This function relates the likelihood of some psychophysical judgment, such as "yes, I see it," to a measure of stimulus strength, such as light level. Early in the last century, a few researchers advocated that an analogous function would be valuable for memory research (Ernst, Smith, Moessner, Rudisill, \& Atwater, 1924; Williams, Titchener, \& Boring, 1918). Recently, we (Zhou, Kahana, \& Sekuler, 2004) introduced such a function, which we called a mnemometric function.

To understand what a mnemometric function is, imagine that some stimulus is presented and remembered. If the subject's memory is probed with that exact same stimulus, there is high probability that it will be recognized. So over many trials, the proportion of recognition responses, $\mathrm{P}(\mathrm{Yes})$, to that probe will be high. However, if memory is probed with a stimulus that is very dissimilar to the remembered stimulus, $\mathrm{P}($ Yes $)$ will be low. Between these two extreme cases there are intermediate casesprobes of varying levels of similarity to the original stimulus. And these probes should evoke varying, intermediate values of $\mathrm{P}(\mathrm{Yes})$.

The mnemometric function, examples of which are shown in Figure 3, is a kind of snapshot of memory strength. It relates a subject's recognition responses to the similarity between a probe and one or more study items. As the probe varies (or "roves") along some perceptual dimension, the responses it evokes on multiple trials trace out variation in memory strength. The mnemometric functions shown in Figure 3 come from a study (Kahana, Zhou, Geller, \& Sekuler, 2007) in which two study-item gratings were presented on each trial. Although the spatial frequencies of the gratings varied from trial to trial, the difference between study items was constrained: Sometimes the items were separated by two just-noticeable differences (panels A and B), other times they were separated by eight just-noticeable differences. 
The mnemometric functions shown in Figure 3 demonstrate several important features of recognition memory. First, the clear bimodality seen in panels $\mathrm{C}$ and D demonstrates that the study items are represented in memory as exemplars rather than in some aggregate form. Second, the spread of responses around each mode indicates the variability associated with the exemplars. This variability makes it impossible to discern bimodality in panels $A$ and $B$, where study items were very similar to one another. Third, the relative advantage in memory enjoyed by the study item that is presented last (the recency effect) produces near-mirror-image relationships between the functions in panels $\mathrm{C}$ and D. The higher and lower modes seem to swap locations between the panels because the relative spatial frequency of the first and second study items was reversed. On some trials (panel C), the second study item had higher spatial frequency than the first study item; on other trials (panel D), the reverse was true. In both cases, the mode associated with the second study item is higher than it is with the first study item. Fourth, these mnemometric functions - and others as well—show no evidence of systematic shift in average memory away from the actual spatial frequencies of the study items. Finally, the blue lines in each panel show predictions made by NEMo. When NEMo was compared to various alternative models, we (Kahana et al., 2007) found that NEMo provided a far better fit to the mnemometric functions than did either standard global matching models or models in which recognition decisions are based only on the single study item most similar to the probe.

\section{FUTURE DIRECTIONS}

Despite the successes of our stimulus-oriented approach to memory, much work lies ahead. Whereas current modeling efforts have been limited to fitting data from individual lists, the human brain is able to focus retrieval on one target list while still retaining some information learned on previous lists. In other words, memory's slate may not be wiped entirely clean after each trail. To address this, models will need to incorporate a mechanism for coding memories within their temporal context and using contextual information to target specific memories. Also, we expect that mnemometric functions will find additional uses, both theoretical and applied. For example, because they afford rich information about similarity-based confusions in recognition memory, mnemometric functions could be a valuable tool for a quantitative assessment of eyewitness testimony, including lineup identification. Another exciting challenge is to identify the neural circuits that compute summed similarity. This effort could start by correlating brain signals measured with noninvasive electroencephalographic (EEG) recordings or functional magnetic resonance imaging methods, with model-derived values of summed similarity and homogeneity on each trial of an experiment. Finally, advances in computational methods will enable us to analyze and compute similarity relations for complex natural stimuli. For example, new computational techniques, operating on large digital databases of potential stimuli, could enable researchers to apply similarity-based models to verbal materials. These and other developments promise to extend far beyond the laboratory the power of a stimulus-oriented approach to memory.

\section{Recommended Reading}

Clark, S.E., \& Gronlund, S.D. (1996). Global matching models of recognition memory: How the models match the data. Psychonomic Bulletin \& Review, 3, 37-60. A review and comparison of memory models, including global matching models.

Estes, W.K. (1994). Classification and cognition. Oxford: Oxford University Press. A clearly written, user-friendly introduction for readers who wish to expand their knowledge of basic issues and approaches to classification and related cognitive functions such as recognition.

Kahana, Zhou, Geller, \& Sekuler (2007). (See References). A representative application of a stimulus-oriented approach to memory, including comprehensive tests of alternative accounts.

Pasternak, T., \& Greenlee, M.W. (2005). Working memory in primate sensory systems. Nature Reviews Neuroscience, 6, 97-107. A review of findings on memory for sensory stimuli and of the contribution of various brain regions to such memory.

Sekuler, R., McLaughlin, C., Kahana, M.J., Wingfield, A., \& Yotsumoto, Y. (2006). Short-term visual recognition and temporal order memory are both well-preserved in aging. Psychology \& Aging, 21, 632-637. An example of how a stimulus-oriented approach can be applied to age-related changes in memory.

Acknowledgments-Supported by National Institutes of Health grants MH068404, MH55687, and MH61975. Many thanks to Kristina Visscher, Feng Zhou, Grace Hwang, Yuko Yotsumoto, Aaron Geller, Shivakumar Viswanathan, and Marieke van Vugt for contributions to the research presented here.

\section{REFERENCES}

Ashby, F.G., \& Maddox, W.T. (1998). Stimulus categorization. In A.A.J. Marley (Ed.), Choice, decision, and measurement: Essays in honor of R. Duncan Luce (pp. 251-301). Mahwah, NJ: Erlbaum.

Bogacz, R., \& Brown, M.W. (2003). Comparison of computational models of familiarity discrimination in the perirhinal cortex. Hippocampus, 13, 494-524.

Ernst, J.L., Smith, F.E., Moessner, L.R., Rudisill, E.S., \& Atwater, M.J. (1924). Further data for an associative limen. American Journal of Psychology, 35, 255-261.

Kahana, M.J., \& Sekuler, R. (2002). Recognizing spatial patterns: a noisy exemplar approach. Vision Research, 42, 2177-2192.

Kahana, M.J., Zhou, F.A., Geller, A., \& Sekuler, R. (2007). Lure-similarity affects visual episodic recognition: Detailed tests of a noisy exemplar model. Memory \& Cognition, 35, 1222-1232.

Nosofsky, R.M., \& Kantner, J. (2006). Exemplar similarity, study list homogeneity, and short-term perceptual recognition. Memory \& Cognition, 34, 112-124. 
Stevens, S.S. (1951). Mathematics, measurement, and psychophysics. In S.S. Stevens (Ed.), Handbook of experimental psychology (pp. 149). New York: J. Wiley \& Sons.

Visscher, K., Kaplan, K., Kahana, M.J., \& Sekuler, R. (2007). Auditory short-term memory behaves like visual short-term memory. PLOS, Biology, 5, e56.

Williams, H.D., Titchener, E.B., \& Boring, E.G. (1918). Minor studies from the psychological laboratory of Cornell University: XL. On the calculation of an associative limen. American Journal of Psychology, 29, 219-226.

Yotsumoto, Y., Kahana, M.J., Wilson, H.R., \& Sekuler, R. (2007). Recognition memory for realistic synthetic faces. Memory \& Cognition, 35, 1233-1244.

Zhou, F., Kahana, M.J., \& Sekuler, R. (2004). Short-term episodic memory for visual textures: a roving probe gathers some memory. Psychological Science, 15, 112-118. 